\title{
Copycat Fast Casual Restaurants: Consumer Response of Dublin Professionals 25 - 39
}

\author{
Audrey Brewer \\ MSc Marketing \\ Department of Business, Dublin Business School \\ Dublin, Ireland
}

(c) Audrey Brewer. This work is licensed under the Creative Commons Attribution-NonCommercial-Share Alike 4.0 International License. To view a copy of this license, visit https://creativecommons.org/licenses/by-nc-sa/4.0/ .

\section{Abstract}

The fast casual market in Ireland is growing at a rate three times that of other restaurant categories. It is especially popular with young professionals aged 25-39, representing more than one third of their food spend. Many Dublin restaurateurs have taken this opportunity to capitalise on the success of existing restaurant brands and open "copycat" eateries within the capital. These restaurants appropriate concepts, menus and trade dress from leader brands outside Ireland, often to commercial success. By using a mixed quantitative and qualitative approach, this research aims to explore Irish consumer perception and patronage of five Dublin copycat restaurants and negotiate the results of this with consumers' evaluation of authenticity. Two hundred and twenty-seven questionnaire responses were evaluated and phenomenologically assessed through in-depth interviews to try to explain the socioeconomic and cultural factors of these results. Findings indicate a generally positive acceptance towards copycatting in Ireland, while still evaluating the "original" restaurant as better in most categories. In this context, a moderate similarity approach appears to work best when the leader brand is not present. Respondents did not have an overall negative view of copycats, and even fewer reported a desire to stop visiting such restaurants once informed about them. Interviews investigated the regulatory, economic and cultural underpinnings for this, suggesting consumer response is dictated by contextual factors that are unique to the Irish commercial landscape.

Keywords: fast casual, casual dining, copycat branding, Irish cuisine, food marketing

\section{Introduction}

Fast casual eateries are experiencing a boom in Dublin, particularly within the young professional market segment. Dubliners aged less than 39 years spend more money at fast casual restaurants than on groceries, and the market segment is growing three times faster than other restaurant types (Bord Bia, 2015). As a burgeoning category, 
fast casuals contain a specific set of consumer expectations. Because of their recent definition, however, fast casual research is markedly underdeveloped.

The Franchise Times places fast casuals between casual dining and quick service, with higher check averages ( $€ 7$ - 15), an adult demographic, "upscale decor", and payat-the-counter service (2008). This list is non-exhaustive but helped create parameters for defining the Dublin copycats included in this study.

Research has suggested that fast casual restaurants cultivate their loyalty mainly through trust and value, with trust being of more importance than value (Chang, 2013). This speaks to the power of a great branding strategy as a way to cut corners on other restaurant attributes. Further, trust cultivated by prominent leader brands could explain how copycats are able to "ride coattails" into similar success. Because trust and authenticity are often linked, it will be important to see whether consumers evaluate the two similarly. Previous research by Kuo-Chien Chang (2013) suggests adding innovative features to a restaurant's overall branding strategy can aid in the perception of trust and authenticity. This technique does not appear to be fully utilised by copycats in Dublin, so new research could show its usefulness in this context. Bufquin, Partlow and DiPietro's (2015) study found while food, service and atmosphere were important for urban restaurant patrons, social and health concerns were not. Another key finding noted the largest disparity between the importance of good quality food and resulting satisfaction, meaning customers desired better food than they received in this category.

There does not appear to be an extant review of copycat restaurants, nor of copycat branding's relationship with Irish consumers. Copycats are better documented in the fast-moving consumer goods sector, with over $50 \%$ of grocery store brands estimated to copy a leader brand's appearance (Morton and Zettelmeyer, 2004). Femke van Horen's variance analysis study suggests, in FMCG, moderate similarity copies perform better than high similarity when a leader brand is available (2010). However, when a leader is absent, the higher similarity will win. This research also found for consumers, a "buying" mindset prefers a high similarity copy, while a "judging" mindset has the opposite effect. This speaks to a consumer's flexible judgment capabilities, depending on the purchase context they are currently in. In studies where both product packaging and quality are considered, consumers punish the copycat whose quality belies its appearance (Campbell and Kirmani, 2000). Intentional deceit is a major source of dissatisfaction for consumers; Dublin's fast casual copycats possess a crucial advantage of existing where their leader brand is not in the consumer's "choice set." This means many consumers have not tried the leader brand offerings, and therefore cannot directly compare the two. This research aims to discover if copycat restaurants are reviewed better because of this.

The competitive edge provided by original leader brands is short-lived and unpredictable (Lieberman and Montgomery, 1988). Copycats present a disadvantage to leaders, as it proves impossible to bar them from entering the market - particularly in food service. In published reports of copycat restaurants, venues in Boston and New York were "named and shamed" by news outlets yet remained opened and 
operational. Few restaurateurs bring menus, recipes or trade dress to a patent or trademark office, and rarely mandate non-disclosure agreements for recipes (Hamersley, 2015). Even when these measures are taken, it is the restaurant owner's responsibility to pursue legal action - an expensive and tedious endeavour, made more difficult when considering international borders (Hamersley, 2015). This would appear to contribute to the influx of copycats that operate solely on the Irish isle, isolated from leader brands.

To the researcher's knowledge, there are no news articles discussing Irish copycat restaurants - save, ironically, the case of restaurateur Niall Fortune, owner of Irish restaurant chain Eddie Rocket's. In 2013, Fortune successfully barred a franchisee from using his restaurant's tagline, colors and signature jukeboxes for their restaurant Rockin' Joes (Deegan, 2013). What is not mentioned in the article is that Eddie Rocket's own features were appropriated from American chain Johnny Rocket's in the 1980s - effectively making Eddie Rockets a copycat itself. The absence of discussion on this topic in Ireland is stark considering how many copycat restaurants exist. This appears to reinforce restaurant copycatting as a normalised practice in the country.

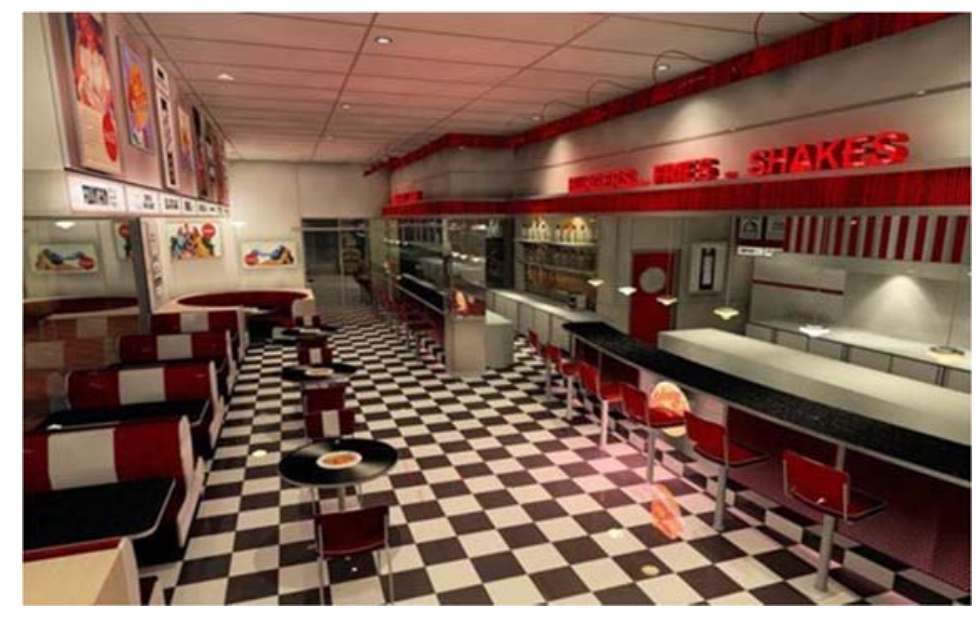

Figure 1: Johnny Rockets Interior, New Jersey 


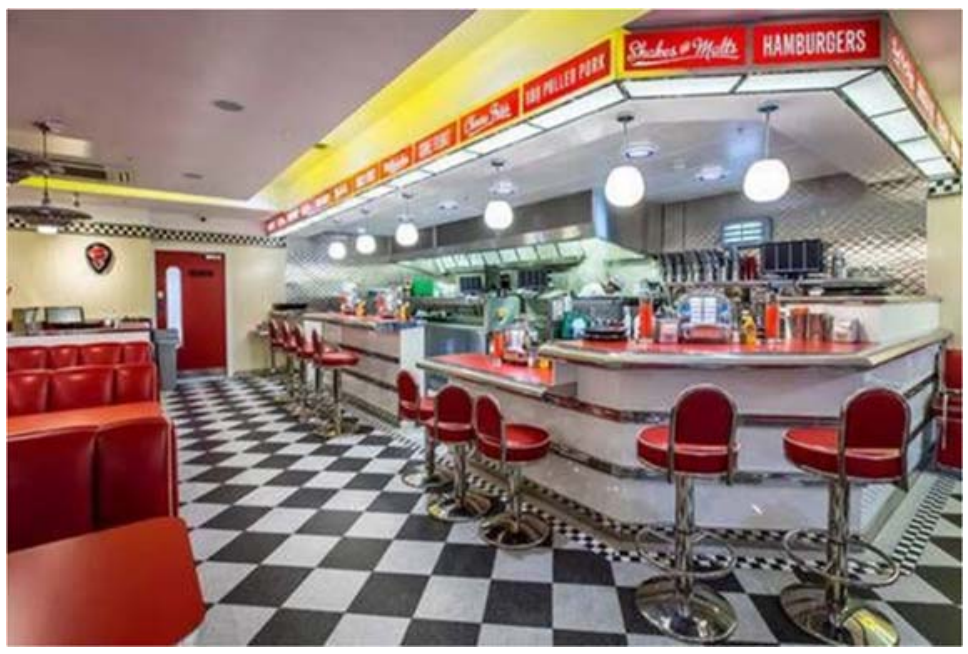

Figure 2: Eddie Rockets Interior, Dublin
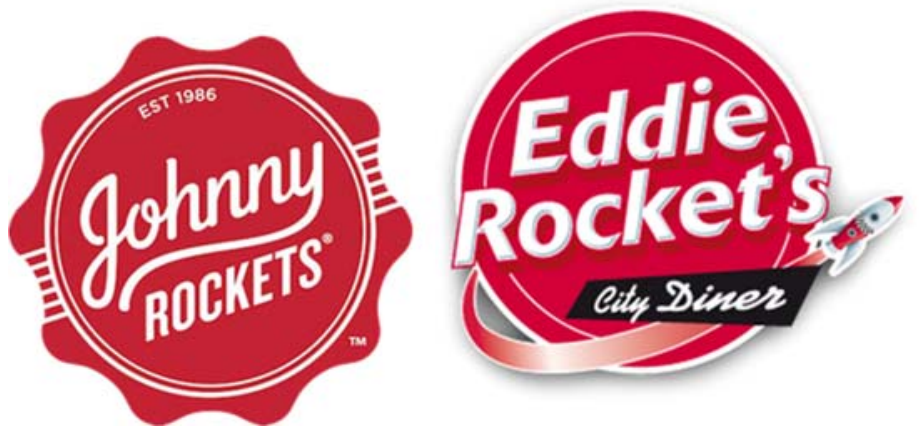

Figure 3: Logo Comparison

Rather than positioning authenticity as a top-down ethos, many brands manipulate token authentic elements to appeal to consumers (Gilmore and Pine, 2007). One such example comes from American smoothie shop Jamba Juice, who grow wheatgrass instore to symbolise a "commitment to real nutrition" (2007). When performing research for this paper, it was found that Jamba Juice used the art of cartoonist David Rees in marketing campaigns without attribution later that year. This kind of tokenised authenticity raises the question of committing to authentic practices on a variable basis. It raises an important question of how consumer perception changes in the face of contradictory brand tactics such as these.

Any cuisine displaced from its country of origin will be a modification, formatted to fit another culture. This perspective will be explored when speaking with Irish culinary experts. There is an historical link between authenticity and 'Irishness' which may explain how residents interpret these concepts. Irish authenticity was traced by Neil O'Boyle in research into what defines an "Irish" advertisement (2009). One question raised was the concept of Irishness itself, which O'Boyle concludes is more uncertain 
than ever. O'Boyle warns that becoming entrapped in "authentic narratives" can alienate a brand from reaching new audiences. If culture is defined as "the way we do things" as one marketer described, Ireland's culture is certainly changing - but into what? O'Boyle states the solution to this problem must be "to avoid the petrification of tradition, as well as the multinational Los Angelisation of society" (2009). A city's restaurant landscape undoubtedly contributes to this cultural debate, and copycat restaurant evaluation could provide a pertinent cross-section of that perspective.

Food authenticity is top of mind in Irish food marketing, noted as a consumer trend to watch by Kim Hogan of the Irish Food Board (2017). A stroll around their headquarters in Dublin reflects a similar sentiment. Their 2017 reception area showcased streetfacing displays centred on authentic ideals. Icons of culinary provenance - wooden fruit boxes and hand-painted signs - dress the windows, as well as photographs of organic farms, seeming to define the personality of the nation's food economy. When speaking to experts, it will be valuable to see if such representation matches their personal experience.

Copycat research highlights the malleability of consumer behavior and judgment. All the while, restaurateurs experience little protection against copycats - even more so in Ireland than the US. This research field would value from fresh insight, as research suggests benefits and drawbacks to the copycat method. Precisely how to "copy well" is something this research aims to elucidate. In a city that has played cosmopolitan catch-up since the 1980s, Dublin copycat restaurants appear to fill consumer needs in a time-sensitive, high-demand environment. Understanding what works best in this context is crucial to succeeding in an increasingly competitive commercial landscape.

\section{Methodology}

This research sought to answer the question: How do Dublin professionals 25 - 39 perceive and patronise copycat fast casuals, and how does this relate to their valuation of authenticity? It was determined that the most comprehensive way to answer this query was via a mixed methods research approach. While existing works have relied on either a qualitative or quantitative approach towards restaurant evaluation and copycat branding, few have incorporated both styles. Combining methodologies will not only provide a baseline perspective but will elucidate the sociocultural motivations behind its responses.

A stratified sampling frame was developed using two cluster groups. These clusters came from two Dublin food businesses: the fast casual restaurant Token, and Dublin delivery service Lunch Team. Both mailing lists included a significant amount of the target population (full-time employed persons between 25 and 39) and were in some form related to the fast casual samples in the survey. 272 vetted responses formed the base of this data. Of this, 123 responses came from an email list for Token, while 159 came from Lunch Team. These responses allowed for a $6 \%$ margin of error with a $95 \%$ confidence level. The average age of participants was 31 . 
Analysis of consumer responses required rigorous use of models to reflect trends and test hypotheses, including OLS regressions, cross-tabulation graphs, and expression of interquartile data. The central framework for this study was the DinEX model developed by Antun et al. in 2010. The DinEX is a detailed scale of over 20 restaurant attributes, which the researcher found untenable when asking consumers to evaluate up to six samples. Evaluations of food, service, and atmosphere were kept for this study, and health and social valuations were removed to accommodate factors of trustworthiness, authenticity and good value. The DinEX's 4-point rating scale remained, as did their two-factor questioning of both 1) importance and 2) satisfaction.

Six restaurants were evaluated - five copycats, and one "control" non-copycat to compare results. The restaurant Token was deemed an "original" control because the author assisted in its development and could therefore confirm it did not include copycat branding methods. The copycats (Eddie Rocket's, Eathos, Pitt Bros., Boojum, and Chopped) were chosen as each restaurant had discussion about its copycat status online, and shared objective similarities with brand leaders. Not each restaurant functions as a carbon copy of a leader brand; some have differentiated by adopting new logos or trade dress. A variety of appropriation levels was hoped to elucidate why some copycats perform better than others.

Qualitative investigation sought to answer the why beneath the quantitative results and was performed via in-depth interviews with industry experts. Each expert was selected on a case-by-case basis. Two of the interview candidates were selected for their current experience with Dublin fast casual entrepreneurship. It was thought they could provide a firsthand account into contemporary consumer tastes, as well as insight into the logistics of opening a Dublin food business. The other two interview candidates were selected for their academic background in Irish food studies, paired with their previous career experience in the industry. These interviews were anticipated to provide a more historical tracing of Irish culinary trends and patterns over time.

Table 1: List of Interviewees

\begin{tabular}{|c|c|c|c|}
\hline Interview A & Interview B & Interview C & Interview D \\
\hline $\begin{array}{c}\text { Professor of } \\
\text { Cookery and Irish } \\
\text { Culinary History, } \\
\text { Former Career } \\
\text { Chef }\end{array}$ & $\begin{array}{c}\text { Professor of Food } \\
\text { Studies, Former } \\
\text { Food \& Hospitality } \\
\text { Manager }\end{array}$ & $\begin{array}{c}\text { Non-copycat Dublin } \\
\text { restaurant owner, } \\
\text { Businessman }\end{array}$ & $\begin{array}{c}\text { Non-copycat Dublin } \\
\text { restaurant owner, } \\
\text { Chef }\end{array}$ \\
\hline
\end{tabular}




\section{Results}

The perception of Dublin copycats by consumers may be categorised as "good." The 6 evaluated attributes ranked near to each other in the good range, with slight variance among interquartiles. In terms of patronage rates, copycats appeared popular. Sixty eight percent of respondents had visited at least 3 of the 5 fast casuals before, and only $20 \%$ of consumers held a negative view of the overall practice. The consumer's relationship to authenticity, as anticipated, raised many questions. While consumers identified the original restaurant as more authentic than the copycats, overall assessment of copycat authenticity remained good. Only in the most overt cases of copycatting was a restaurant rated as "Fair" in this attribute (Eddie Rockets, Mdn 2).

\section{Key Findings:}

- Authenticity ranked lowest in terms of importance in restaurant attributes, and consumers generally rated copycats highly in terms of authenticity satisfaction. However, consumers reported a full Likert point difference between the importance and assessment of Food Quality in copycats, indicating this is an area for improvement for such restaurants.

- The original restaurant Token scored a full Likert point higher than the best performing copycat (moderate similarity Boojum) and earned a median 'Excellent' score in four distinct categories: Authenticity, Food Quality, Style and Trustworthiness.

- Considering the success of the moderately similar and the original restaurant in the survey, it would indeed appear innovative and unique menu items assist with a restaurant's appeal. The moderately similar copycat performed better than a high similarity copycat even though leader brands were not present for the study. Further, a consumer's past experience with a leader brand did not affect their assessment of a copycat.

An appreciation for original details was reiterated by the Irish consumers' preference for a moderate similarity approach, which integrated some original qualities into an already successful service concept. This feeds into a broader question of the modern Irish self-concept, as investigated by O'Boyle (2009). Fears of Dublin adopting a mono-cultural position to become like other cities appear frequently, from protests over Starbucks' city centre permeation (Paul, 2017) to ongoing debates on building height restrictions (O'Sullivan, 2016). This may explain why a differentiation approach works better than carbon copies for Dublin restaurant brands.

Another interesting factor in the copycat evaluation comes from observing the two survey clusters. Responses coming from the Token email list (the original restaurant) indicated a stronger negative response to copycatting. 35\% of its consumers disapproved, compared to $16 \%$ of delivery service email respondents. This disparity brings up an interesting distinction between consumer judgment and preference. It appears an overt relationship with an "original" brand leads to a stronger judgment against copycatting. Interestingly, while the two clusters' approval of copycatting differed, their individual assessments of each restaurant failed to show much disparity. 
Therefore, the impetus remains on the perception of authenticity and originality, rather than any tangible indicators of a behavioral shift.

In-depth interviews backed up this sentiment. Interviewee A described the modern consumer as one who expects choice and self-identifies as "discerning," yet alongside this self-conception is a lack of awareness of what actually happens inside restaurant kitchens. They attributed importance to a perception of quality and authenticity, rather than any tangible reality. Likewise, Interviewee B believed the food preferences of the demographic were highly influenced by the aesthetics of social media culture. This content, he posits, contributes to a consumer's love for food storytelling, but does not perform the heavy lifting required to inspire a real dedication to or knowledge of food provenance. However, both agreed that the fast casual food category is popular within the demographic and remains successful despite these shortcomings.

All interviewees acknowledged the perceived importance of food authenticity - not just to consumers, but from the Irish Food Board's chosen food messaging. However, they also shared a certain distaste for the term, as well as expressing doubts for any way of consumers quantifying it:

"I don't like the word authentic because I don't think there is such a thing." - Interviewee B

"Quality is valued highly by consumers, but it's just a perception. Authenticity stops at the counter." - Interviewee A

Interviewee B went so as far to say they don't believe authenticity is truly possible in a fast casual category, let alone in copycats. He attributes this to the fundamental nature of fast casual operations, citing examples of visiting fast casuals and asking to speak with the chef, only to find many fast casuals did not have staff who self-defined as such. To a career chef (Interviewee B), this lack of culinary experimentation, management or quality control effectively limits the fast casual's ability to produce an authentic product at all, regardless of its branding origins. Interviewee $D$, a chef behind a local sustainable breakfast hub, agrees that authenticity "doesn't really matter" to Irish consumers. It's a choice his restaurant has made because it is something he believes in, but he acknowledges his consumers don't actually know the source of his ingredients and is likely a case of perception that earns their patronage.

"At the moment the buzzwords are all about 'local, sustainable.' As a food historian, I say 'Here we go again." - Interviewee B

The cyclical nature of food trends was also addressed in the interviews. A historian of Irish food, Interviewee B states the current trend of authenticity and provenance is more likely a trend than a radical psychological shift. Embracing the locality of food, he states, first appeared as a trend in the nouvelle cuisine movement of the 1970s and has re-entered the discussion of Irish food culture many times. While he believes 
"nothing is new," he does see some form of innovation in fast casuals, in that many provide "something that is uniquely rich" - a signature high-calorie menu item that separates their menu from competitors. He notes it is difficult to get restaurant funding for a concept that has not been tested in Ireland, which puts a damper on restaurants' ability to innovate. Yet, Interviewee A found that the life cycle of Dublin food trends remains faster than ever and supplying some form of "exotic non-homogenous fare" is vital to capturing public interest. Further, it appears consumers with an overt relationship to an "authentic and original" brand lead to a stronger judgment against the act of copycatting. For this consumer segment, highlighting a brand's authentic and innovative qualities could be an effective approach to gaining a more positive perception. Gilmore and Pine's (2007) suggestion of adding smaller authentic or innovative elements, like a weekly special, may be sufficient middle ground between copycatting and innovative branding based on the data received.

Legislation was cited as a potential trouble area for restaurateurs looking to open a food business. This "red tape nightmare" (Interviewee A) could help explain the prevalence of copycat restaurants in Dublin, as copycats are often owned by serial entrepreneurs with experience in quick, efficient restaurant openings. Interviewee A believes that the Vintners' Association, the publican advocacy group, is the main impediment to a better restaurant culture in Dublin; "not quite cartel, but close". The ability for a restaurant to sell alcohol under a full license in Ireland is exceedingly difficult, as pub licenses are made available on a fixed-number, bidding basis rather than case-by-case application. Interviewee A cites a similar license in the UK being available for less than 1,000 GBP, while Irish equivalent bids start at 100,000 EUR. Original restaurant owner Interviewee $C$ reports his restaurant opening was pushed back six months in order to obtain and finance the transfer of a full pub license, a timeline that can be prohibitively expensive when accounting for operational costs like city centre leaseholds. This can discourage restaurants from pursuing the license at all, which hospitality expert Interviewee A finds vital to a "complete" dining experience. This has led most Irish consumers to divide their evening between restaurant and pub, which may explain the popularity of a "quick in, quick out" fast casual offer.

This "punitive" legislation (Interviewee A) towards restaurants selling alcohol appears to be a contributing factor to the copycat phenomenon in Ireland. For serial entrepreneurs who prioritise expediency and the bottom line, it appears more logical to open a fast casual that can fit into a portion of a consumer's leisure time rather than try to retain customers for an entire evening. Many original/leader restaurant brands from outside Ireland are aware of Irish regulations and choose to not expand into the market for this reason (Interviewee A). Brands will sometimes provide the ability to franchise their offering, but this is not always the case. Knowing leaders are reluctant to enter the market, yet aware that Irish consumers are receptive to fast casuals, a space opens for copycat restaurants to fill.

The legislation barring restaurants to alcohol licensing appears directly related to the popularity of drink culture in Ireland. The Vintner's Association is reported as having a 
longstanding advantage through its lobbying efforts (Interviewee B), and the government's prioritisation of pubs is treated as de facto for the restaurant industry.

“Foreigners don't open Irish restaurants. They open Irish pubs." - Interviewee A

As a leisure activity, drinking often takes precedence over food consumption in Ireland. "I love the pub," says Interviewee D, "and I think part of why we're drawn to fast casuals is we can fit it in between the pubs on a night out." It appears culturally in Ireland, food functions as a practical necessity, while drinking is a socialising pleasure. While the overall interest in food is increasing in Dublin as the city grows, a lingering preference for pub over restaurant may explain the Irish consumers' general satisfaction with copycats.

One positive contribution found in multiple interviews is the fast casual's accessibility to a wide range of consumers. Their price point makes them more democratic than the "white tablecloth" dining that long dominated the Irish restaurant market (Interviewee B). Many experts view this as a benefit, as it brought the young demographic "out of the pub" and interested in food culture more than before. Fast casual owners, Interviewee D and Interviewee $C$, see this casualisation of the dining experience as a generally beneficial trend - it gives consumers the ability to experiment with foods that were previously inaccessible. However, the demand to provide diversity and value in a casual environment may inhibit the full potential for quality innovation in Dublin's restaurant market. As seen in the next theme, there are additional factors that prevent many from entering the market at all.

Interviewees $D$ and $C$ posit that from a consumer standpoint, the economic state of young Dublin professionals may contribute to the prioritisation of value in the restaurant survey (Mdn 4 - Very Important). While salaries have increased by less than $5 \%$ in most sectors in the past year, the cost of renting in Dublin has risen by $15 \%$ (CSO, 2011). This naturally leads to less disposable income for consumers. When combined with a general priority of pubs over restaurants as an evening experience, the importance of good value elevates further.

In the case of Dublin copycats, a disparity exists between the consumers' priority of food quality and resulting satisfaction, which some interviewees attributed to the nature of fast casual operations. Such restaurants rarely hire chefs to train cooks, finesse recipes, or source better-quality ingredients, all of which could help improve their Food Quality ranking. The exception to the fast casuals in this survey is Token, who employs a full-time chef who both introduces specials and continually trains employees. Token scored a "4 - Excellent" in Food Quality during the survey and won Dublin Restaurant of the Year at the Irish Food Awards in 2017, indicating a positive consumer response from this approach. The prioritisation of Food Quality may have the ripple effect of improving other attribute categories, such as Authenticity \& Trustworthiness. While authenticity may not be worthwhile to focus on arbitrarily, there 
appear to be advantages to taking a food quality focus where authenticity is an intrinsic component.

Qualitative insights are grounded in the internal structure of Dublin's restaurant legislation and culture, which reflect how consumers engage and maintain relationships with copycats. The same authenticity response - namely, that it functions as perceptual "lip service" rather than cornerstone of assessment (Interviewee A) remained consistent across the quantitative and qualitative findings.

It is evident that there are cultural and economic reasons for the copycat phenomenon in Ireland, and that the consumers' responses fit inside these environmental factors.

Generally speaking, it appears consumers desired better food quality than they received in Dublin copycat fast casuals. Innovative or authentic details helped a restaurant's appeal, with the moderately similar restaurant performing best of the copycats. This indicates a Dublin consumers' desire for differentiation, while not completely discouraging the copycat practice.

Indeed, past experience with a copycat did not impact assessment, and valuations of authenticity often did not seem to correlate with copycat status. As bolstered by both survey and interviews, authenticity in the broader ideological sense was not considered an important concept. This suggests an open interpretation into its application for consumers.

While the original restaurant was met with high consumer ratings, its startup costs were increased due to punitive legislative and financial processes that revealed themselves in interviews. These factors ultimately suggest a prioritisation of the pub industry in the capital to the detriment of budding restaurateurs. This seems to permit copycat restaurateurs with large starting capital to enter a space where international leader brands or independent business owners cannot or do not fill.

\section{Recommendations}

While a copycat offering can in many ways be labelled objectively, assessment of its authenticity is far more fluid. If a restaurant copies another brand's menu and food operations, but then prioritises food provenance and local sourcing, these effects may even out their assessment by consumers. In many of the discussions, the consumer's core issue appears to be a devalued dining experience rather than copycatting itself. As seen in the consumer survey results, if a fast casual is to engage in copycatting, they must match or improve its quality to gain acceptance.

Based on the qualitative interviews, it appears the punitive relationship between restaurants and governmental bodies places restrictions on a more diverse fast casual landscape. Loosened regulation on full alcohol licenses, as well as measures towards further municipal rent control would be a welcome addition for the Dublin restaurateurs interviewed for the study. As proposed by Interviewee B, creation of an official union for Irish chefs could also be useful, as it would potentially curtail the migratory drain which sees many chefs leave Ireland for better training and higher wages. While there 
is no clear way to prevent copycat restaurants from entering a market, providing easier routes for independent entrepreneurs in the restaurant sector could add quality and variety to Dublin's culinary landscape.

\section{Conclusion}

This study focused on the Irish fast casual industry and the position of copycat brands within the market. The fast casual sector is a rapidly growing dining category in Dublin which contributes not only to the economy, but the cultural fabric of the capital. One key conclusion showed that regarding copycat restaurants, patrons desired a betterquality experience than what was received - particularly when compared to the "authentic" restaurant. What the study was unable to prove, however, was a direct benefit of authenticity for authenticity's sake within Dublin restaurant branding. In moments where authenticity had a direct impact on a guest's restaurant experience for instance, through higher quality ingredients - the benefits of authenticity became evident. However, other spaces emerged that painted authenticity's importance with broader brushstrokes. For instance, it did not appear from interviews that consumers had much idea of the true nature of the ingredients they were being served or who was preparing it, nor did consumers report outright distaste for the idea of copycatting in general. Rather, an integration of token authentic elements seemed to satisfy when it was applied in a meaningful way for consumers.

Another key takeaway from this study stemmed from the socioeconomic and legislative barriers faced by restaurant business owners in Dublin. Such factors have likely led to the spread of sub-optimal copycat restaurants. While the fast casual restaurant industry has grown in recent years, the scene has become increasingly competitive and expensive for entrepreneurs to join. This has led to an increased presence of large restaurant groups laying claim to multiple food brands and businesses, some containing copycats. The large starting capital of such groups enables them to perform serial restaurant openings at a time-sensitive rate. With economic barriers like a lack of funding for innovative concepts (Interviewee D), high municipal rents, and cost-prohibitive alcohol licenses, access to the Dublin restaurant industry has created a narrow and challenging funnel for entrepreneurs to pass through. Increased attention to this oligopolistic trend would likely allow for more creative and bespoke restaurant concepts to emerge, and a more "authentic where it matters" restaurant landscape in the city.

This study focused on fast casual restaurants and the position of copycat brands in the market. The fast casual industry plays an increasingly vital role in the Irish culinary landscape and provides a large amount of revenue to the State from both tourists and Irish residents. One key conclusion from this research is that the quality expectations of Irish residents are not currently being met.

This research was performed with the intention of understanding the Irish consumer's relationship to authenticity within a restaurant branding context. Such interest was borne from the researcher's experience developing the video game arcade restaurant Token. Token opened in Dublin in July 2017 after nearly two years of research and 
development. It was a financial and commercial success upon opening and seemed to reinforce a previously untapped demand for the kind of nostalgia experience this restaurant concept provides.

Towards the end of the research, the author learned a new fast casual restaurant was set to open in Cork. It went from conception to launch in under three months, funded and owned by a large Irish entertainment group. From this new restaurant's menu design and arcade game selection, down to the small canvas bags used to hold their "signature tokens," some similarities struck the researcher as familiar. The restaurant's name differed, yet oddly, was shared with an arcade in Canada. This revelation personally confirmed the researcher's belief in the perennial, inevitable nature of restaurant copycatting in Ireland. It served as an apt metaphor for the unpredictability of being a leader brand, and to the researcher, justified a market investigation into this practice.

\section{References}

Antun, J.M., Frash Jr, R.E., Costen, W., and Runyan, R.C. (2010) Accurately assessing expectations most important to restaurant patrons: The creation of the DinEX Scale, Journal of Foodservice Business Research, 13(4), pp. 360-379.

Bord Bia (2015) 'Irish Foodservice Market Worth €6.3 billion'. Available at: https://www.bordbia.ie/corporate/press/2015/pages//rishFoodserviceMarketWorth\%E2\%82\% AC63billion.aspx (Accessed: 20 April 2017).

Bufquin, D., Partlow, C.G. and DiPietro, R.B. (2015) 'Measuring restaurant patrons' perceptions and expectations: An importance-performance analysis using the DinEX model', Journal of Foodservice Business Research, 18(3), pp. 226-243.

Campbell, M.C. and Kirmani, A. (2000) 'Consumers' use of persuasion knowledge: The effects of accessibility and cognitive capacity on perceptions of an influence agent', Journal of Consumer Research, 27(1), pp. 69-83.

Chang, K.C. (2013) 'How reputation creates loyalty in the restaurant sector', International Journal of Contemporary Hospitality Management, 25(4), pp. 536-557.

CSO Ireland (2011) 'CD108: Population by Sex, Province County or City, Age Group and Census Year'. Available at:

https://www.cso.ie/px/pxeirestat/Statire/SelectVarVal/Define.asp?maintable=CD108\&PLangu age $=0$ (Accessed: 31 May 2017).

Deegan, G. (2013) 'Eddie Rocket's lawsuit settled for €250,000', The Irish Times, 23 October [Online]. Available at:

https://www.irishtimes.com/business/retail-and-services/eddie-rocket-s-lawsuit-settled-for250-000-1.1569824 (Accessed: 1 December 2017). 
The Franchise Times (2008) 'What exactly is fast casual?', The Franchise Times, 1January [Online]. Available at: https://www.franchisetimes.com/January-2008/What-exactly-is-fast casual/ (Accessed: 1 December 2017).

Gilmore, J., and Pine, J. (2007) Authenticity: What Consumers Really Want. Boston: Harvard Business School Press.

Hamersley, G. (2015) 'Among chefs, sharing's OK, stealing isn't', The Boston Globe, 21 October [Online]. Available at: https://www.bostonglobe.com/lifestyle/2015/10/21/amongchefs-sharing-stealing-isn/DuTcPVtd3TJBk3cpQhAWOL/story.html (Accessed: 2 December 2017)

Hogan, K. (2017) 'Top 10 Consumer Trends for 2017'. Bord Bia. Available at: https://www.bordbia.ie/industry/manufacturers/insight/alerts/pages/toptenconsumertrendsfor 2017.aspx (Accessed: 10 Sept 2017).

Horen, F.V. (2010) 'Breaking the mould on copycats: what makes product imitation strategies successful?' Kli Dissertations Series. Tilburg: CentER, 18, pp.110-123.

Lieberman, M.B. and Montgomery, D.B. (1988) 'First-mover advantages', Strategic Management Journal, 9(S1), pp. 41-58.

Morton, F.S. and Zettelmeyer, F. (2004) 'The strategic positioning of store brands in retailermanufacturer negotiation', Review of Industrial Organization, 24(2), pp. 161-194.

O'Boyle, N. (2009) 'Knowing what it means to be Irish: experience as practice in the advertising industry', Social Identities, 15(5), pp.565-584.

O’Sullivan, F. (2016) 'Should Dublin Build Higher?'. CityLab. Available at: https://www.citylab.com/equity/2016/06/should-dublin-build-higher/485078/ (Accessed: 23 October 2017).

Paul, M. (2017) 'Dublin Starbucks coffee protest ran to a script worthy of 'South Park". The Irish Times, 8 September [Online]. Available at: https://www.irishtimes.com/business/retailand-services/dublin-starbucks-coffee-protest-ran-to-a-script-worthy-of-south-park-1.3213172 (Accessed: 23 October 2017). 